\title{
“Breaking Bad News" Current Practices in Hospitals
}

\author{
Brig Syed Arsalan Haider Bukhari* \\ Department of Orthopedics, Northern Punjab Rawalpindi, Pakistan
}

Submission: February 25, 2018; Published: March 19, 2018

*Corresponding author: Brig Syed Arsalan Haider Bukhari, Department of Orthopedics, Northern Punjab Rawalpindi, Pakistan, Email: bonemender541@gmail.com

Abstract

Objective: To evaluate the current practice of breaking bad news to patients and their relatives in Hospitals and to identify the weaknesses in this practice in light of International standard.

Study design: A cross sectional study, through convenience non-probability sampling.

Material and methods: The sample size consisted of Administrators of Tertiary Care $(n=20)$, specialists of Tertiary Care hospitals ( $n=40)$, Ortho-oncology patients of these hospitals $(n=60)$ and their relatives $(n=40)$. All the people in sample were given structured questionnaire and the inferences were drawn based on the responses given.

Results: The results indicated that majority of administrators had not set up any patient doctor communication setups in their hospitals. Most of the specialists were sharing the disease related matters with the patients and their relatives, without any prior preparation.

Conclusion: The findings highlighted a persistent major void in this crucial aspect of patient doctor communication .The encouraging aspect was the awareness by all the stake holders of this deficit and a will to initiate proper structured and certified training on the subject.

Keywords: Illness; Patient doctor communication; Oncology counselling; Bad news

\section{Introduction}

Health and disease go hand in hand in a human's life. Whenever a person is brought down by any ailment, his life comes to an effective halt. Many diseases are trivial and transient in nature but some are grave and chronic, even threatening the life of the effected patient. In such a situation, the most taxing duty for a doctor is to communicate the bad news to the effected patients and their relatives, thus indicating the importance of an effective communication in today's health care. "Bad news" in its literal terms is self-explanatory, but in technical terms it is any information that results in a cognitive, behavioural or emotional deficit in the person receiving the news.

Studies have shown that contrary to what many doctors had thought in the past, most of the patients want to be told the truth about their condition by the treating physician. They want to understand their diagnosis, estimate their prognosis in relation to what treatments they will be offered and which options they may choose to pursue these goals. Breaking-badnews encounters are more challenging than one might think. Many factors such as un-predictable emotional reaction of the patient and his relatives, their literacy level, extent of knowledge about the disease, emotional status before receiving the bad news, and physician's own awareness of his standing in regards to performance of this sensitive task, are the common reasons which usually deter the doctor himself, from delivery of bad news.

In our health care system the problems of lack of patients understanding, poor literacy levels, social compulsions, misinterpreted religious dictates, language barriers, highly charged emotional atmosphere and superficiality in patient doctor interaction make the situation more difficult and the task of breaking bad news an uphill one. Critical evaluation of the present state of practice, quality and methodology of breaking bad news in our setup and determining the existing pitfalls which might be belittling the overall efforts being extended to the patients by health care providers, is mandatory. Steps in right direction will help bolster medical care setup and the confidence and motivation of health service providers, thereby uplifting our present medical services.

\section{Materials and Methods}

A cross sectional study was carried out through convenience non-probability sampling. The study on practice of breaking bad news to orthopedic patients with bone tumours admitted in tertiary care hospitals was conducted over a period of six months from October 2012 to March 2013. The patients and their relatives were randomly involved in a questionnaire-based 
approach. The data was collected at the end of the period and analyzed in light of responses given by the participants to the questions in the questionnaire. The sample size consisted of health care administrators of Tertiary Care hospitals $(n=20)$, specialists of Tertiary Care hospitals $(n=40)$, patients of Tertiary Care $(n=60)$ and the relatives of the effected patients $(n=40)$. The data was obtained through structured questionnaire. The questions in the survey were also asked as interview from the patients and their relatives who were not literate. All the results of both the questionnaire and the interview were then analyzed in the light of the objectives designed and inferences were drawn based on percentage dominion on SPSS version 18.

\section{Results}

The results were analyzed to assess the attitudes and importance of practice attached by the administrators and specialists in the sample hospitals towards effective breaking of bad news to the patients and their relatives. The hospital administrators $(n=20)$ were included in the study. $100 \%$ hospital administrators had no structured and internationally compatible operating procedure in vogue in their hospitals regarding breaking or communicating bad news to the patients. $78 \%$ were aware that their specialist was breaking the bad news to the patients and $22 \%$ acknowledged that this was being done by the nursing staff. $83 \%$ had no infrastructure for patient doctor communication and only $30 \%$ had taken a few steps like to promote patient doctor communication. 19\% had started training of the doctors, $11 \%$ had held workshops but $70 \%$ still had done nothing to promote or implement patient doctor communication.

Interpretation of the data received revealed that the specialists $(n=40)$ were communicating bad news to the patients and their relatives in a manner not comparable to any internationally standardized model (Spikes model of Buckman). $65 \%$ felt a need to break a bad for more than 5 times / week but $90 \%$ of such specialists performed this task based on selfdevised methods. 94\% had never received any formal certified training on the subject. $62 \%$ specialists were sharing the disease related matters with the patients and their relatives but in a self-devised method. $90 \%$ of respondents performed the duty on self-generated methodologies without any consistency in performance. $69 \%$ respondents were unaware of the importance of venue for breaking bad news.

$74 \%$ specialists tried to communicate with frequent usage of medical language which was difficult by the patients and their relatives to comprehend or fully understand, thereby completely undermining the mere purpose of such information transfer. $69 \%$ specialists displayed lack of emotional intelligence and continued with the information transfer in an apathic manner instead of stopping the dialogue till the clientele settled down emotionally. Only 40\% respondents discussed the future line of action with the patients. The confidence level of the specialist was also very low and $44 \%$ specialists were wary of unexpected reaction from clientele. $63 \%$ had average comfort levels during breaking of bad news. $81 \%$ of the respondents reported of having experienced confrontation with clientele due to absence or improper breaking of bad news to them. $100 \%$ specialists wanted initiation of a proper structured and certified training on the subject, to improve their healthcare outcomes. $18 \%$ specialist completed the duty in piecemeal information transfer manner.

The overall common flaws in current practice of breaking bad news to patients and their relatives inferred from the above mentioned data revealed, absent official protocols, absence of will to implement proper practice, inadequate Knowledge about International models of breaking bad news, non-standardized local protocols, absence of proper training in regards to patient doctor communication, poor environment selection, poor comfort level during communication, poor preparation before breaking bad news, lack of systematic delivery of information, usage of medical terms, minimal emotional intelligence, noticeable lack of Empathy and above all lack of any concrete steps to eradicate this major flaw of day to day practice. There was a noticeable strong feeling of hidden frustration among most patients and their relatives indicating a major deficiency regarding the practice of breaking bad news to the clientele, thereby producing a decline in patient confidence in the doctors and subtly generating an overall dissatisfaction.

\section{Discussion}

Concealment of a bad news has always been in the nature of humans. Pages of history tell us that in the courts of the Kings, the bearer of bad news usually met with an adverse end. Thinking on similar lines the physicians of the past also believed in masterly concealment of a bad diagnosis or treatment outcome. In ancient times it was considered to be evil and cruel to inform a patient of a serious disease or terminal outcome. Any physician indulging in the act of breaking a bad news was subjected to stern disciplinary action on grounds of violation of human sensitivities [1]. Hippocrates doctrine stands a testament to this observation, which states that, conceal most nasty things from the patient while you are attending to him. Even in face of being in knowledge.

Of the fact that the patient will eventually die of his disease, give necessary orders with cheerfulness and serenity revealing nothing of the patient's future or present condition to him, for many patients will take a turn for the worse...by forecast of what is to come. [2]. However as the world evolutes with its experiences and learning, the priorities and methodologies also changed in this regard. The advent of realization of human rights and the promulgation of World Health Organization's "Universal Declaration of Human Rights" [3,4] adopted in 1948, (article 25) traditional paternalistic models of patient care have given way to an emphasis on patient autonomy and empowerment. This was followed by latest legislations like the "European charter of Patient's rights" [5] which outlined the basic patients' 
rights currently at risk. These included: the right to preventive measures, the right to access to information, the right to consent, free choice, privacy and confidentiality, respect of patients' time, observance of quality standards, safety, innovation, avoidance of unnecessary suffering and pain, avoidance of preferential personalized treatment, and the right to complain and to receive compensation.

These rights are also linked to several international declarations and recommendations, issued by both the WHO and the Council of Europe. Health professions council of South Africa in their "National patients' rights charter" article 2.2 also states, "Every citizen has the right to participate in the development of health policies, whereas everyone has the right to participate in decision-making on matters affecting one's own health" [6]. Such changes in stance regarding patient's rights and his "right to know", acutely focused on the fact that the concealment practice be abolished forthwith and the patient is given full insight into the matters which directly and adversely affect his or her future and life. This coupled with acute rise in the basic medical knowledge of a common man with a massive influx of information through the advances in technology resulted in major shift in policies of international healthcare setups where the patients are now considered the sole owners of information relating to their health [7]. Withholding of such information from the affected patient and his care givers, is considered as professional misconduct and violation of basic human rights.

Fifty years ago, most physicians were able to avoid discomfort by concealing the truth from patients, justifying this with the claim that the truth would be too distressing for the patients. By the late 1990 s, $90 \%$ of physicians told patients if they had cancer (Buckman). Now in many countries there are legal and ethical obligations to tell the patients any details about their disease as per their wish [8]. Conveying bad news is a skilled communication. A properly structured and well-orchestrated communication has a positive therapeutic effect [9]. This is a process of information exchange between patient and physician. Proper exploitation of patient's fundamental insight is vital as much as is the knowledge of what not to do and what to expect, because the patient is the one who knows what is hurting him most and he is the one who knows how to move forward [10]. Truthful disclosure of psychologically painful information not only hurts the patient and their relatives but also embarrasses the doctor [11].

A number of related studies have documented such a physician-patient communication, as mostly less than optimal. The main reasons for a physician's tendency to avoid the task of breaking bad news are lack of skills and the reluctance to deal with the patient's or his relatives' feelings. Physicians and nurses typically miss the full range of concerns of patients. Feelings of uncertainty fear, anger, depression and blame are common reactions if bad news is broken poorly [12]. Unfortunately most of the physicians feel incompetent and are afraid of unleashing a negative reaction from the patient or their relatives. Many are reminded of his or her own vulnerability to terminal illness, and they feel powerless over their own and patient's emotional unrest [13].

Lack of proper and expert training in breaking bad news is a handicap to most physicians and health care workers. Having a detailed knowledge about the most important barriers / deterrence or hurdles to an effective breaking of bad news , how to approach the patient and his relatives and what to do and what not to do are the key assets which a doctor must possess before embarking on this extremely important as well as sensitive duty [14]. The common factors which prevent a doctor from delivering the bad news to the patient or his relatives himself, include: Lack of knowledge of patients insight, lack of good rapport with the patient, cultural and social values, language barrier, use of medical language, clientele educational level, emotional state, feeling of guilt and helplessness, poor communication skills training and above all lack of empathy $[15,16]$.

When we consider breaking bad news, we have to follow certain universal principles. Firstly it is important to realize that, the skills which are required to deliver bad news are teachable and therefore are learnable. Second, delivering bad news is always an act which is uncomfortable and marred with a significant degree of uneasiness [17]. Thirdly, learning to deliver bad news thoughtfully methodically and in a structured manner enhances trust between the doctor and his patient and makes good to the total medical care extended. In order to achieve this aim many working models of breaking bad news have been proposed. These models evolved progressively keeping in mind the various requirements of breaking bad news and moved from the basic WHO model to most widely and internationally accepted and practiced Spikes model [18].

The "S.P.I.K.E.S" protocol provides a straightforward and an easy to adopt set of principles for a successful session for communication of bad news. It also suggests ways to assess the situation as it evolves and respond constructively to patients. Show of empathy, evaluating the patient's understanding and acceptance of what he or she has just come to know, and appreciating and respecting that patient's feelings can provide much-needed support to the patient [19]. It also provides an essential psychological support for managing distress and helping the patient face the treatment decisions ahead. The SPIKES protocol effectively achieves these goals. It stands for Setting, Perception, and Invitation. Knowledge, Empathy and Strategizing. Proper adherence to this protocol and adhering to its methodology is the essence to a complete, honest and ethical communication of bad news to the patient and their relatives [19]. The validating, exploratory, and empathic responses should also help you to support the patient, an essential psychological intervention for distress [20]. 


\section{Conclusion}

There is no doubt that a bad news will always remain a bad news and nothing can change its nature, however one thing is changeable for sure and that is the way we communicate it. Sincere efforts to overcome our shortcomings in executing an effective patient doctor communication and an effort by all the stake holders in this context, is the need of the hour. It will immensely improve the quality of healthcare being extended and satisfaction level of our dependent clientele.

\section{References}

1. Percival T (1849) Medical ethics; or a code of institutes and precepts, adapted to the professional conduct of physicians and surgeons. Manchester, England 1803: 166.

2. In Jones WH (1923) (Hippocrates. Decorum, XVI) Hippocrates with an English Translation. Heinemann, London, UK.

3. Walter F Bailea, Robert Buckmanb, Renato Lenzia, Gary Globera, Estela A Beale, et al. (2000) SPIKES--A six-step protocol for delivering bad news: application to the patient with cancer. Oncologist 5(4): 302-311.

4. Wise TN, Dellemonache PM, Bachawati MM (2012) The psychosomatic interview. Adv Psychosom Med 32: 19-34.

5. Munoz Sastre MT, Cooper, Sorum PC, Bernstien, Mullet E (2011) Breaking bad news: the patient's viewpoint. Health Commun 26(7): 649-655.

6. Bou Khalil R (2013) Attitudes, beliefs and perceptions regarding truth disclosure of cancer-related information in the Middle East: A review Palliat Support Care 11(1): 69-78.

7. Buckman R (1992) Breaking Bad News: A Guide for Health Care Professionals. Johns Hopkins University Press, Baltimore, USA, p. 15.

8. Burgers C, Beukeboom CJ, Sparks's L (2012) How the doc should (not) talk: when breaking bad news with negations influence patients' immediate responses and medical adherence intentions. Patient Educ Couns 89(2): 267-273.

This work is licensed under Creative Commons Attribution 4.0 License DOI: 10.19080/OROAJ.2018.11.555808
9. Adeleye AO, Fatiregun AA (2012) Is breaking of bad news indeed unacceptable to native Africans? A cross-sectional survey of patients in a Nigerian neurosurgical service. Acta Neurol Scand 127(3): 175-180.

10. F Van Dalen J, Bonke B, Van Weel-Baumgarten EM, Brouwers M, Grosfeld F, et al. (2012) Teaching and training in breaking bad news at the Dutch medical schools: a comparison. Med Teach 34(5): 373-381.

11. Fields SA, Johnson WM (2012) Physician-patient communication: breaking bad news. WV Med J 108(2): 32-35.

12. Rosenzweig MQ (2014) Breaking bad news: a guide for effective and empathetic communication. Nurse Pract 37(2): 1-4.

13. Simmenroth-Nayda A, Alt-Epping B, Gágyor I (2013) Breaking bad news - an interdisciplinary curricular teaching-concept. GMS Z Med Ausbild 28(4): Doc52.

14. Schildmann J, Schwarz C, Schildmann E, Klambeck A, Ortwein H, et al. (2011) Truth at the bedside. Dtsch Med Wochenschr 136(15): 757761.

15. Narayanan V, Bista B, Koshy C (2010) Breaks Protocol for Breaking Bad News. Indian J Palliat Care 16(2): 61-65.

16. Arbabi M, Roozdar A, Taher M, Shirzad S, Arjmand M, et al. (2010) How to break bad news: physicians' and nurses' attitudes. Iran J Psychiatry 5(4): 128-133.

17. Ishaque S, Saleem T, Khawaja FB, Qidwai W (2010) Breaking bad news: exploring patient's perspective and expectations. J Pak Med Assoc 60(5): 407-411.

18. Tuffrey-Wijne I (2013) A new model for breaking bad news to people with intellectual disabilities. Palliat Med 27(1): 5-12.

19. Shaw J, Dunn S, Heinrich P (2012) Managing the delivery of bad news: an in-depth analysis of doctors' delivery style. Patient Educ Couns 87(2): 186-192.

20. Antoun J, Saab BR (2015) A culturally sensitive audiovisual package to teach breaking bad news in a Lebanese setting. Med Teach 32(10): 868-869.

\section{Your next submission with Juniper Publishers will reach you the below assets}

- Quality Editorial service

- Swift Peer Review

- Reprints availability

- E-prints Service

- Manuscript Podcast for convenient understanding

- Global attainment for your research

- Manuscript accessibility in different formats

( Pdf, E-pub, Full Text, Audio)

- Unceasing customer service

Track the below URL for one-step submission https://juniperpublishers.com/online-submission.php 